\title{
Weak catch bonds make strong networks
}

\author{
Yuval Mulla ${ }^{1,2}$, Mario J Avellaneda ${ }^{1}$, Antoine Roland ${ }^{1}$, Lucia Baldauf ${ }^{1,3}$, Sander J Tans ${ }^{1,3 *}$, Gijsje H \\ Koenderink $^{1,3 *}$ \\ ${ }^{1}$ Living Matter Department, AMOLF, Amsterdam, The Netherlands \\ 2 Institute for Biological Physics, University of Cologne, Cologne, Germany \\ ${ }^{3}$ Department of Bionanoscience, Kavli Institute of Nanoscience Delft, Delft University of Technology, \\ Delft $2629 \mathrm{HZ}$, the Netherlands \\ *Correspondence to: g.h.koenderink@tudelft.nl, tans@amolf.nl
}

Molecular catch bonds are ubiquitous in biology and well-studied in the context of leukocyte extravasion ${ }^{1}$, cellular mechanosensing ${ }^{2,3}$, and urinary tract infection ${ }^{4}$. Unlike normal (slip) bonds, catch bonds strengthen under tension. The current paradigm is that this remarkable ability enables cells to increase their adhesion in fast fluid flows $s^{1,4}$, and hence provides 'strength-on-demand'. Recently, cytoskeletal crosslinkers have been discovered that also display catch bonding ${ }^{5-8}$. It has been suggested that they strengthen cells, following the strength-on-demand paradigm ${ }^{9,10}$. However, catch bonds tend to be weaker compared to regular (slip) bonds because they have cryptic binding sites that are often inactive ${ }^{11-13}$. Therefore, the role of catch bonding in the cytoskeleton remains unclear. Here we reconstitute cytoskeletal actin networks to show that catch bonds render them both stronger and more deformable than slip bonds, even though the bonds themselves are weaker. We develop a model to show that weak binding allows the catch bonds to mitigate crack initiation by moving from low- to high-tension areas in response to mechanical loading. By contrast, slip bonds remain trapped in stress-free areas. We therefore propose that the mechanism of catch bonding is typified by dissociation-on-demand rather than strength-on-demand. Dissociation-on-demand can explain how both cytolinkers ${ }^{5-8,10,14,15}$ and adhesins ${ }^{1,2,4,12,16-20}$ exploit continuous redistribution to combine mechanical strength with the adaptability required for movement and proliferation ${ }^{21}$. Our findings provide a mechanistic understanding of diseases where catch bonding is compromised ${ }^{11,12}$ such as kidney focal segmental glomerulosclerosis ${ }^{22,23}$, caused by the a-actinin-4 mutant studied here. Moreover, catch bonds provide a route towards creating life-like materials that combine strength with deformability ${ }^{24}$.

Here we exploit the actin-binding protein a-actinin-4 and its K225E point mutant, associated with the heritable disease kidney focal segmental glomerulosclerosis type $1^{22,25}$, to identify the role of catch bonds in the mechanical properties of actin networks. Actin networks are key determinants of cell mechanics, together with other cytoskeletal proteins. To isolate the role of catch bonds in actin mechanics, we reconstitute actin networks from purified components. We first characterized the binding affinity of the two protein variants for actin 
filaments in the absence of mechanical load. Co-sedimentation of the crosslinkers with actin filaments (methods) revealed that the K255E mutant has a nearly 10-fold higher affinity $\left(15.55 \pm 0.04{\mu \mathrm{M}^{-1}}^{-1}\right)$ for actin than wild type a-actinin-4 (1.95 $\pm 0.04 \mu \mathrm{M}^{-1}$, Fig. $\left.1 \mathrm{e}\right)$. Fluorescence recovery after photobleaching measurements of crosslinker dissociation confirmed that wild type a-actinin-4 has a substantially higher off-rate than the mutant (Extended Data Fig. 2), consistent with prior measurements in cells ${ }^{26,27}$.

\section{a-actinin-4 forms a weak catch bond while the K255E mutant forms a strong slip bond}

It has previously been speculated that force activates a cryptic actin-binding site of a-actinin4 , based on the crystal structure ${ }^{28,29}$. It was furthermore proposed that the cryptic actin binding site is constitutively exposed by the K255E point mutation, increasing the binding affinity of a-actinin-4 but also abrogating its catch bond behavior (Fig. 1a-b) $)^{13,14,26,28-30}$. To directly test this idea, we tethered single a-actinin-4 molecules to polystyrene beads via DNA handles (2500 base pairs), and probed their binding to fluorescently tagged actin filaments, which fully coated another set of beads (Fig. 1c, see Methods). Using optical tweezers, we trapped an a-actinin-4-coated bead and an actin-coated bead, as verified by simultaneous fluorescence imaging (Extended Data Fig. 3b) and performed bead approach-retraction cycles. When we detected a force increase upon retraction, which indicated a binding event, we subsequently maintained the tether at a pre-set force until the force suddenly dropped to zero and the beads separated (Fig. 1d), indicating forced crosslinker unbinding. The bond lifetime for the wild type a-actinin-4 showed a load dependence consistent with catch bond behavior: short lifetimes at low loads, peaking at an intermediate load (around $4 \mathrm{pN}$ ), and decreasing for further increasing loads (Fig. 1f, red data). By contrast, the K255E point mutant showed slip bond behavior, with a lifetime higher than the wild type variant at low loads (consistent with the biochemical data) and monotonically decreasing for increasing tensions (Fig. 1f, blue data). The single-molecule data provide direct proof of earlier speculations that $\alpha$-actinin-4 forms weak catch bonds whilst the K255E point mutant forms strong slip bonds ${ }^{13,14,26,28-30}$. 

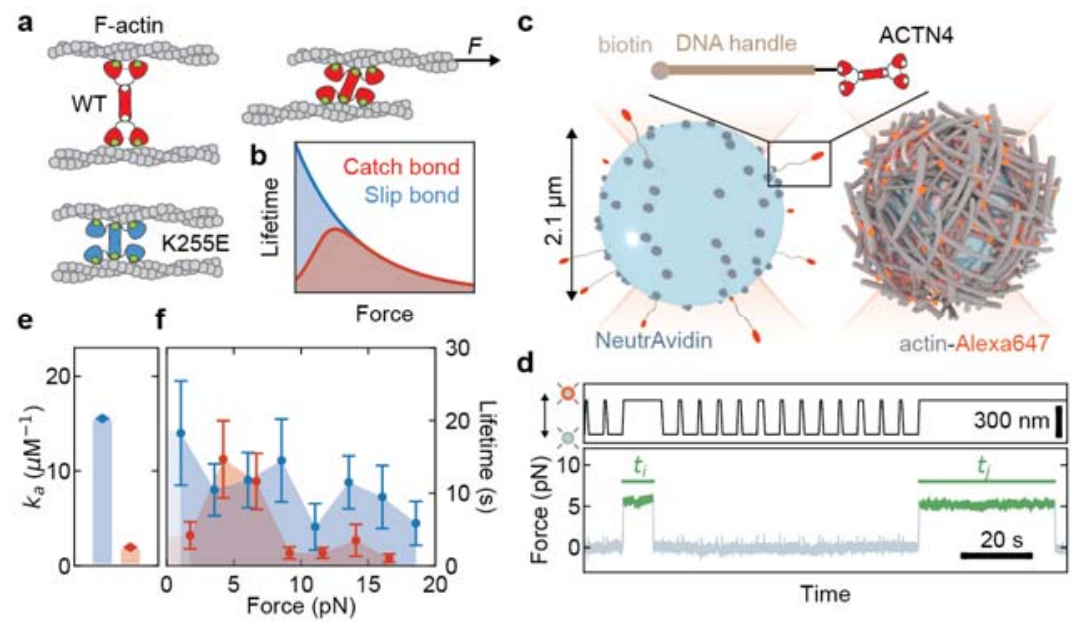

Fig. 1: Single-molecule measurements of actin filament binding reveal catch bonding for wild type $\alpha$ actinin-4 but not the K255E mutant. a, Each monomer of the dimeric crosslinker a-actinin-4 (red) has two weak binding sites for actin filaments (green) and one strong binding site (white) that needs to be activated by force for the wild type (WT) protein (red) whereas it is always exposed for the K255E mutant (blue). b, Conceptually, the actin-bound lifetime of a-actinin-4 should be lower than that of K255E at low force, but the lifetimes should become comparable as force increases. c, Single-molecule force spectroscopy assay, where a crosslinker-coated and an actin-coated bead are trapped using optical tweezers. d, Example trace illustrating the approach-andretract protocol to establish bonds between the crosslinkers and actin filaments (top panel). An increase in the force while retracting indicates the presence of a tether (green), and the lifetime is measured until the instant the tether breaks $\left(t_{i}, t_{\text {}}\right.$, bottom panel). e, Actin association affinity $k_{\mathrm{a}}$ of $a$-actinin-4 (red) and K255E (blue) measured in a co-sedimentation assay. Error bars indicate standard error extracted from a fit assuming Michaelis Menten kinetics (Extended Data Fig. 1a-c). f, Average lifetime of tethers as a function of applied force, as measured by optical tweezers (see panel d). The lifetime of wild type a-actinin-4 (red) initially rises, peaks at a force of $\sim 4 \mathrm{pN}$, and then decreases, as expected for a catch bond. The K255E mutant shows an overall decreasing lifetime, typical of a slip bond. Error bars indicate standard error. Affinity and force spectroscopy data were obtained at 25 ${ }^{\circ} \mathrm{C}$.

\section{Catch bonds increase actin network strength}

The observation that catch bonds are weaker than slip bonds raises the question whether they also form weaker networks. To test the strength of crosslinked actin networks, we copolymerized actin with either crosslinker between the cone and plate of a rheometer and linearly increased the mechanical load (shear stress) in time by rotating the cone until the network ruptured (Fig. 2b, open circles). We simultaneously recorded the applied load (stress) and resulting network deformation (strain) by superposing a small oscillatory shear on top of the stress ramp to measure the differential elastic modulus. We first dissect the effect of bond affinity on network rupturing by measuring networks crosslinked by the mutant slip bonds at either high or low temperature (resp. $25{ }^{\circ} \mathrm{C}$ for low affinity and $10{ }^{\circ} \mathrm{C}$ for high affinity). Consistent with intuition, we find that weaker linkers yield weaker networks (rupture stresses of $6.5 \pm 0.5 \mathrm{~Pa}$ and $8.1 \pm 1.1 \mathrm{~Pa}$ at $25^{\circ} \mathrm{C}$ and $10{ }^{\circ} \mathrm{C}$, respectively, Fig. $2 \mathrm{~b}$, blue vs. 
black bars). At the same time, the weaker networks are more deformable, meaning that they reach a much larger strain before rupturing (63 $\pm 4 \%$ and $129 \pm 10 \%$, resp. Fig. 2 b, blue vs. black bars). So how about the catch bonds, which have a lower affinity than the mutant slip bonds but exhibit a different load dependence? Strikingly, networks crosslinked by the aactinin- 4 catch bonds at $10^{\circ} \mathrm{C}$ were more deformable than either of the slip bond networks (rupture strain of $221 \pm 16 \%$, Fig. $2 b$, red bars) yet also stronger (rupture stress of $24.5 \pm 2.7$ $\mathrm{Pa})$.

How can catch bonds escape the trade-off between strength and deformability that is inherent in normal (slip) bonds? To answer this question, we developed a minimal model where the crosslinked actin network was represented by an array of $N$ reversible bonds sharing a load $\sigma$ (Fig. 2c, see Methods). We assumed nearest-neighbor load sharing (Methods Eq. (2)), which provides a simple yet accurate way to model crack initiation in viscoelastic materials, which is the rate-limiting step of rupturing (Extended Data Fig. 5) 31,32 . We used idealized Bell-Evans force-dependent unbinding kinetics (Methods Eq. (1)) $)^{33}$, and allowed for unbound linkers to rebind at a random new location ${ }^{32,34}$. We chose our parameters in accordance to the force spectroscopy and biochemical data, such that the catch bonds are weaker at low force (Fig. 1b, see Extended Data Table 1 for all parameters). Strikingly, the simulations also showed that weak catch bonds collectively make networks that are stronger than slip bond networks (rupturing at nearly twice the stress, Fig. 2d), yet more deformable (with 10-fold more bond turnovers before rupturing, Fig. 2d). This difference persisted when including partially bound crosslinkers to account for the fact that a-actinin is a homodimer (Extended Data Fig. 6d, Supporting Information). The model also confirmed the experimental observation that simply decreasing the bond lifetime while retaining a slip bond response results in weaker networks (Fig. 2b).
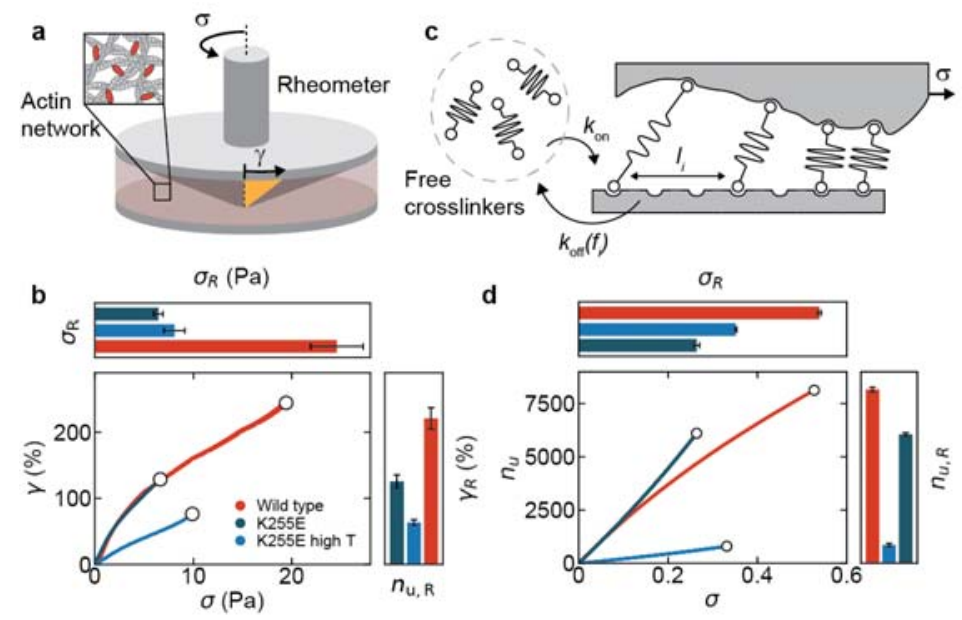
Fig. 2: Catch bonds simultaneously enhance the mechanical strength and the deformability of cytoskeletal actin networks. a, Scheme of rheology experiments to characterize actin network mechanics. We measure the shear deformation $y$ of actin networks crosslinked either with a-actinin-4 or with K255E by linearly increasing the shear stress $\sigma$ in time with a stress rate of $2.0 \mathrm{mPa} / \mathrm{s}$. $\mathbf{b}$, Representative examples of the shear strain $y$ as function of the shear stress $\sigma$ for a-actinin- 4 (red), $\mathrm{K} 255 \mathrm{E}$ (blue), both at $10{ }^{\circ} \mathrm{C}$, and for $\mathrm{K} 255 \mathrm{E}$ at an elevated temperature $\left(25^{\circ} \mathrm{C}\right.$, dark blue) where its lifetime matches that of wild type a-actinin- 4 at $10^{\circ} \mathrm{C}$ (Extended Data Fig. 4c). The white circles indicate the rupture points (see Methods). The top panel shows the average rupture stress and the right panel the average rupture strain for each condition, with error bars representing the standard error ( $N=4$ for each condition). c, Actin networks are modelled as 1D arrays of reversible linkers that stochastically exchange between a bound and freely diffusing state. The applied load $(\sigma)$ linearly increases in time and is shared over all bound linkers proportionally to the distance to the nearest neighbors $l_{i}$. $\mathbf{d}$, The total number of unbinding events per bond $n_{u}$ as a function of applied stress (see Methods), showing the same crosslinker dependence as the rheology experiments. The error bars in the top and right panel show the standard error ( $N=100$ for each condition).

\section{Mechanism of catch bond-induced network strengthening}

To identify the mechanism behind the remarkable mechanical advantage of catch bonds, we quantified the steady state distributions of the load per individual crosslinker (Fig. 3a). At a given macroscopic load, the average force per bond was higher for the catch bonds compared to the slip bonds (resp. $0.241 \pm 0.003$ and $0.223 \pm 0.001$, mean \pm standard error), consistent with their lower bond affinity. Strikingly, however, the distribution of forces for catch bonds was much narrower than for the slip bonds, meaning that slip bond networks contain more bonds that bear high loads. We next tested the size at which gaps initiate cracks in networks of mobile linkers, by ablating adjacent bonds and simulating the network stability as a function of the gap size. Notably, gaps twice as large were required to rupture networks of catch bonds compared to slip bonds (Fig. 3b). These findings suggest that catch bonds 'dissociate-on-demand' from low-stress areas, freeing up crosslinkers that rebind in high-stress areas and hence prevent the initiation of cracks. Simulations showed that the mechanical advantage of catch bonds over slip bonds was indeed lost when the catch bonds are immobile (Extended Data Fig. 6c).

As crosslinker rebinding is important for the mechanism, we next investigate how the mechanical advantage of catch bonding depends on the binding rate. We find that in case of low binding rates, slip bonds provide stronger networks than catch bonds (Extended Data Fig. 7a): in this regime, catch bond-induced dissociation strongly decreases the bound fraction, thereby weakening the network. By contrast, when the binding rate is high, increased dissociation barely affects the bound fraction as crosslinkers rapidly rebind. Therefore, catch bonds provide stronger networks only when the binding affinity is high (binding faster than unbinding, Extended Data Fig. 7a), which is the relevant situation for real actin networks crosslinked by a-actinin-4 (Extended Data Fig. 1d) and also appears to be the 
relevant regime in cells given the strong co-localization of a-actinin-4 with the actin cytoskeleton ${ }^{13,26}$. To experimentally test these predictions, we performed rupturing experiments on actin networks where we increased the bond affinity by decreasing the temperature from $25{ }^{\circ} \mathrm{C}$ to $10{ }^{\circ} \mathrm{C}$ (Extended Data Fig. 4a-c) $)^{34}$. Consistent with the model's prediction, the rupture stress indeed increased more steeply for the a-actinin- 4 catch bonds than for the K255E slip bonds (Extended Data Fig. 7b).

Our model predicts that catch bonding triggered by network stress is key to explain the increased strength of the wild a-actinin- 4 crosslinkers. To test whether the loads exerted on the network were indeed sufficient to activate the catch bonds, we determined the crosslinker unbinding time from the network mechanics at different levels of shear stress, using a small oscillatory stress at different frequencies to measure the viscoelastic response time (Supplementary Information, Methods). This assay provides the characteristic network relaxation time, which is directly proportional to the crosslinker unbinding time ${ }^{35}$. The network relaxation time in case of wild type a-actinin- 4 crosslinkers increased with increasing shear stress, consistent with catch bonding. For the K255E crosslinkers, the stress relaxation time was larger than for the catch bonds at low shear stress but similar at high stress (Extended Data Fig. 4e-h), mirroring the behavior observed at the single molecule level. These findings show that macroscopically applied stresses above $5 \mathrm{~Pa}$ indeed activate strong binding for wild type $\alpha$-actinin-4, whereas the K255E mutant behaves like a conventional slip bond, being strongest at small loads.
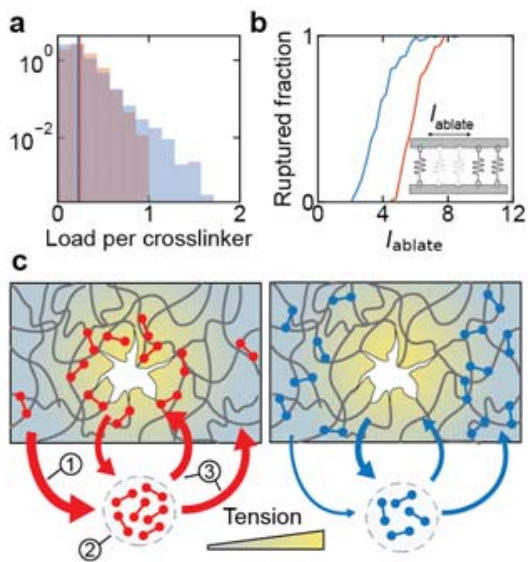

Fig. 3: Simulations reveal that catch bonds strengthen networks by suppressing force inhomogeneities. a, The distribution of forces per bond $f$ measured at steady state. The average bond force (vertical lines, $0.241 \pm$ 0.003 and $0.223 \pm 0.001$, mean \pm standard error) is larger for catch bonds (red) than for slip bonds (blue), but the force distribution is much narrower. $\mathbf{b}$, The fraction of networks that rupture when a gap of varying ablation length $l_{\text {ablate }}$ is introduced for both catch (red) and slip bonds (blue). Inset: schematic of the ablation simulation. c, Selfassembly mechanism explaining the mechanical advantage of weak catch bonds (red, left) over strong slip bonds (blue, right). The thickness of the colored arrows codes for the on- and off-rate of the linkers. 1. Catch bond 
linkers in low tension areas rapidly unbind, increasing the pool of unbound linkers (2). As a result, there is increased binding everywhere in the network (3), at the expense of only the linkers in low tension areas. The net result is that the force distribution homogenizes, preventing crack initiation. By contrast, slip bonds preferentially localize in low-stress areas.

\section{Discussion}

Our work reveals a new role for catch bonds in the cytoskeleton, namely to simultaneously increase its mechanical strength and its deformability. Contrary to the common intuition that catch bonds provide strength-on-demand, our model shows that they make strong networks because dissociation-on-demand enables them to suppress force inhomogeneities and thus postpone crack initiation. Our findings provide a molecular mechanism to explain the low mechanical stability of kidney cells in patients afflicted by heritable disease kidney focal

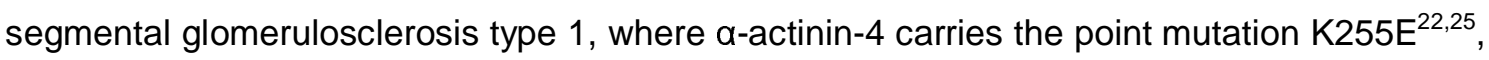
and suggest a similar explanation for other diseases where loss of catch bonding leads to tissue failure, such as Von Willebrand disease $2 \mathrm{~B}^{11,12}$. The generality of our model implies that this same mechanism also applies to catch bonds in cell-matrix and cell-cell adhesions $^{1,2,16-20}$, as dissociation-on-demand reduces friction whilst simultaneously minimizing the risk of complete cell detachment. Therefore, our results suggest that catch bonds are widespread in the cytoskeleton and at cellular interfaces to break this deformability/strength trade-off, and it would be interesting to investigate force-dependent binding of more crosslinkers and adhesins, such as filamin and IgSF CAMs. Finally, our findings offer a cell-inspired route to create hydrogel materials that are strong yet sufficiently deformable for applications in regenerative medicine ${ }^{36}$. Compared to existing strategies such as hierarchic structuring ${ }^{24,37}$, catch bonding is a simpler and more modular approach to improve material strength, as it only affects a single length scale, namely the molecular structure of the linker. Synthetic analogues of catch bonds have recently been discovered and provide an excellent starting point towards highly dynamic yet strong biomimetic materials ${ }^{38}$. 


\section{METHODS}

\section{Computational model}

To investigate the effect of molecular catch bonding on the strength of cytoskeletal filament networks, we use a computational model we recently developed to predict failure of transient networks ${ }^{35}$, using a Gillespie algorithm to model stochastic linker binding and unbinding. We consider a 1D model of $N$ linkers that share an externally applied load $\sigma$ (Fig. 2c). We model the effect of a force $f$ on the unbinding rate $k_{\text {off }}$ of a bound linker $i$ using the Bell-Evans equation ${ }^{39}$ :

$$
k_{\mathrm{off}, i}\left(f_{i}\right)=k_{\mathrm{off}, 0}^{\mathrm{catch}} \cdot e^{\frac{-f_{i}}{f_{1 / e}^{\text {catch }}}}+k_{\mathrm{off}, 0}^{\text {slip }} \cdot e^{\frac{f_{i}}{f_{1 / e}^{\text {sip }}}}
$$

The first exponent models the catching of the weakly bound state, whereas the second exponent models the slipping of the force-activated state. We compare catch bonds with slip bonds, which do not require forceactivation for strong binding $\left(k_{\mathrm{of}, 0}^{\mathrm{catch}}=0\right)$, keeping all other parameters identical (Fig. 1b, see Extended Data Table

1 for the full list of parameters used for each simulation). We define the bond affinity as $K=\frac{k_{\text {on }}}{k_{\text {on }}+k_{\text {off, } 0}}$, where $k_{\text {on }}$ is the on-rate of unbound linkers. To account for the mobility by random diffusion of the linkers after unbinding, we allow for unbound linkers to rebind at a random new location ${ }^{39}$. As the actin concentration is significantly larger than the crosslinker concentration both in our reconstituted networks (resp. $48 \mu \mathrm{M}$ and $0.48 \mu \mathrm{M}$ ) and in living cells (on the order of resp. $100 \mu \mathrm{M}$ and $1 \mu \mathrm{M}^{40}$ ), we consider 10-fold more binding sites than crosslinkers to prevent competition for actin-binding sites. For control simulations where the linkers are immobile (Extended Data Fig. $6 c)$, we only allow for rebinding in the same place where the crosslinker unbound ${ }^{41}$.

It is known that stressed networks connected by reversible bonds exhibit spontaneous crack initiation and propagation due to inhomogeneous load sharing ${ }^{42}$. We reproduce this rupturing behavior using a minimal model where the force per linker $f_{i}$ is proportional to the global applied stress and the distance between its nearest neighbors on both sides $l_{i}$ in 1D (Fig. 2c):

$$
f_{i}=\frac{l_{i}}{\sum_{i} l_{i}} \cdot \sigma \cdot \mathrm{N}
$$

We use a periodic boundary condition to prevent edge effects. We initialize networks by randomly placing $N \cdot K$ linkers (see Extended Data Table 1 for all parameters). The Supplementary Information and Extended Data Fig. $6 a-b$ contain a more detailed discussion of the effect of network size.

We use bond turnover as a proxy for deformability. Although the exact, quantitative relationship is complex and beyond the scope of this work, bond turnover is proportional to actin network deformability ${ }^{35}$. Indeed, actin networks without bond turnover rupture at only approximately $10 \%$ shear strain ${ }^{43}$, whereas the transiently crosslinked networks here reach maximal strains of up to several hundred percent, showing that bond turnover dominates network deformability.

\section{Protein purification}

Human wild type a-actinin-4 and its K255E point mutant were purified as previously described ${ }^{44}$. Briefly, E. Coli cells were transformed to express recombinant crosslinkers with a His $\sigma_{6}$-tag. Induction was performed with $500 \mu \mathrm{M}$ isopropyl $\beta$-D-1-thiogalactopyranoside for eight hours at $25^{\circ} \mathrm{C}$. After centrifugation at $6000 \mathrm{~g}$ for 15 minutes, cells were resuspended in $20 \mathrm{mM} \mathrm{NaCl}, 5 \mathrm{mg} / \mathrm{ml}$ lysozyme and $20 \mathrm{mM}$ HEPES, $\mathrm{pH}$ 7.8. The cells were lysed by a freeze-thaw cycle and the lysate was centrifuged at $20,000 \mathrm{~g}$ for $30 \mathrm{~min}$. The recombinant proteins were purified 
from the supernatant using a QIAGEN nickel column that was first washed with 20 bed volumes of $500 \mathrm{mM} \mathrm{NaCl}$, $25 \mathrm{mM}$ imidazole, and $20 \mathrm{mM}$ HEPES, $\mathrm{pH}$ 7.8. The recombinant proteins were eluted with 10 bed volumes of 500 $\mathrm{mM} \mathrm{NaCl}, 500 \mathrm{mM}$ imidazole, and $20 \mathrm{mM}$ HEPES, $\mathrm{pH}$ 7.8, concentrated using Centricon filters (Millipore), and purified by gel filtration in $150 \mathrm{mM} \mathrm{NaCl}, 20 \mathrm{mM}$ HEPES pH 7.8, and $10 \mathrm{mM}$ dithiothreitol (DTT). Actin was labeled using an Alexa Fluor Labeling Kit purchased from ThermoFisher and biotin-actin was purchased from Cytoskeleton.

To ensure we compare a-actinin-4 and K255E at the same concentration in all our assays, we determined the ratio of the protein stock concentrations by measuring the intensity of the protein bands on an SDS-PAGE gel. We chose this method because, unlike UV-VIS spectrophotometry, it specifically measures the protein of interest and excludes the contribution of any contaminants. The proteins were cysteine-labeled using maleimide-activated Oregon Green at a ratio of five fluorophores for every crosslinker at room temperature for $1 \mathrm{~h}$. Labeled proteins were separated from free dye molecules by gel filtration using a Superdex 200 column (GE Healthcare) ${ }^{44}$.

Actin was purified from rabbit psoas skeletal muscle as described in reference ${ }^{45}$, including a gel filtration step to remove oligomers ${ }^{34}$. The concentration was determined by measuring the optical absorbance at $280 \mathrm{~nm}$. Aliquots were snap-frozen and stored at $-80^{\circ} \mathrm{C}$ in G-buffer (2 mM tris-hydrochloride $\mathrm{pH}$ 8.0, $0.2 \mathrm{mM}$ disodium adenosine triphosphate, $0.2 \mathrm{mM}$ calcium chloride, $0.2 \mathrm{mM}$ dithiothreitol) to prevent polymerization. After thawing, we stored G-actin stock samples overnight at $4 \stackrel{\circ}{\mathrm{C}}$. The next day, we spun the sample at $120000 \mathrm{~g}$ to remove any remaining aggregates. The supernatants were stored at $4{ }^{\circ} \mathrm{C}$ and used within 7 days. We polymerized actin at a concentration of $48 \mu \mathrm{M}(2 \mathrm{mg} / \mathrm{ml})$ in an F-buffer consisting of $50 \mathrm{mM} \mathrm{KCl}, 20 \mathrm{mM}$ imidazole $\mathrm{pH} 7.4,2 \mathrm{mM} \mathrm{MgCl}_{2}$, $1 \mathrm{mM}$ DTT and $0.5 \mathrm{mM} \mathrm{MgATP}$ in the presence of crosslinker at a concentration of $0.48 \mu \mathrm{M}$ (corresponding to a molar ratio of $1 / 100$ crosslinker/actin and on average around 1 crosslinker for every $0.5 \mu m$ length of actin filament). We verified that the networks under these conditions are isotropic and spatially uniform by confocal fluorescence imaging (Extended Data Fig. 8). Unless otherwise mentioned, all chemicals were purchased at Sigma Aldrich.

\section{SDS-PAGE gel protocol and quantification}

SDS-PAGE gels were used to characterize and quantify purified proteins. In all cases, $20 \mu$ sample was mixed with $20 \mu \mathrm{l}$ InstantBlue and boiled at $95{ }^{\circ} \mathrm{C}$ for 5 minutes in a closed Eppendorf vial. $30 \mu \mathrm{l}$ of this solution was loaded onto a 4-15\% Mini-PROTEAN TGX Precast Protein Gel with 10 wells of $30 \mu \mathrm{l}$. Gels were run for 30 minutes at $200 \mathrm{~V}$, washed with Milli-Q water, stained overnight with InstantBlue and washed three times with tap water. Band intensities were quantified using Image ${ }^{46}$. Background correction was applied to all band intensities by subtracting the average intensity of a region adjacent to the band of interest.

\section{Fluorescence Recovery After Photobleaching}

The bond lifetime of bound crosslinkers was measured via Fluorescence Recovery After Photobleaching (FRAP) using a Nikon A1 confocal microscope with a perfect focus system, 100x 1.40 NA oil immersion objective and $100-\mathrm{mW} 488 \mathrm{~nm}$ argon ion laser. We acquired 10 images to determine baseline fluorescence and then performed photobleaching by increasing the laser power such that $50-70 \%$ of the fluorescence intensity was bleached in 0.5 seconds. We then tracked the fluorescence recovery with a low-intensity beam during a period of approximately 5 times the typical recovery time, with a sampling rate that halved every 10 frames, starting with 10 frames/second. During imaging, the exposure time was kept fixed at 0.1 second/frame. We bleached a circular area of $2 \mu \mathrm{m}$ radius and used an equally sized area as a reference. The laser intensity during imaging was chosen such that the reference intensity dropped less than $5 \%$ during the recovery phase. To extract a timescale for fluorescence 
recovery, $\mathbf{T}_{\mathrm{FRAP}}$, the time-dependent intensity normalized by the intensity of the reference area was fitted with a

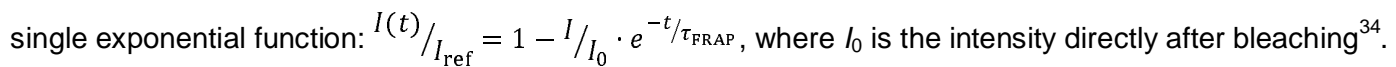

\section{Co-sedimentation assay}

A volume of $25 \mu \mathrm{l}$ monomeric (G-)actin at increasing concentrations was co-polymerized with either $\alpha$-actinin-4 or K255E in F-buffer at room temperature, keeping the crosslinker concentration constant $(0.1 \mu \mathrm{M})$. After two hours of polymerization, the actin network together with the bound crosslinkers was spun down at $120000 \mathrm{~g}$. Afterwards, $20 \mu \mathrm{l}$ was gently pipetted from the supernatant and run on an SDS-PAGE gel as described above. The fraction of bound linkers $\varphi_{\text {bound }}$ was determined by subtracting and normalizing the crosslinker band intensity $I$ at a particular actin concentration by the band intensity $I_{0}$ in the absence of actin using ImageJ: $\varphi_{\text {bound }}=\frac{I-I_{0}}{I_{0}}$.

\section{Rheology}

Rheology was performed using a stress-controlled Kinexus Malvern Pro rheometer with a stainless-steel coneplate geometry having a radius of $20 \mathrm{~mm}$ and a 1 degree cone angle. We loaded $40 \mu \mathrm{l}$ samples of actin monomers, directly after mixing with either a-actinin-4 or K255E and F-buffer, onto the bottom plate and quickly lowered the cone. A thin layer of Fluka mineral oil Type A was added around the edge to prevent solvent evaporation, and the sample was closed off with a hood to prevent any effects of air flow. Actin polymerization was followed by applying a small oscillatory shear with a strain amplitude of $0.5 \%$ and a frequency of $0.5 \mathrm{~Hz}$. After $2 \mathrm{~h}$ of polymerization, the elastic shear modulus G' and viscous shear modulus G" were measured as a function of frequency by performing small amplitude oscillatory shear measurements at frequencies between $0.01-10 \mathrm{~Hz}$, taking 30 logarithmically spaced data points. Frequencies above $10 \mathrm{~Hz}$ could not be accessed as inertial effects from the rheometer started to dominate the rheological response of the actin network. Finally, we performed a rupture experiment by linearly increasing the stress in time at a constant loading rate $(2 \mathrm{mPa} / \mathrm{s})$ until the network ruptured. The rate of $2 \mathrm{mPa} / \mathrm{s}$ was chosen because it was sufficiently slow to reliably measure the differential storage modulus at every stress level, whilst it was sufficiently fast to prevent network aging effects during the stress ramp. To unambiguously identify the rupture point, we measured the differential elastic modulus of the network as a function of stress by superposing small stress oscillations on top of the stress ramp. We observed stress-stiffening above a certain threshold stress, consistent with prior literature ${ }^{35}$, followed by a rapid drop of the stiffness that signals rupture. We defined the rupture point as the stress value at which the differential storage modulus $K^{\prime}$ peaked (Extended Data Fig. 4d). This approach allowed us to simultaneously identify the rupture stress and rupture strain (Fig 2c).

We determined the stress-dependent bound lifetime of the crosslinkers from the network mechanics, by applying a recently developed biopolymer network mode ${ }^{35}$ to the time- and stress-dependent network elasticity (differential storage modulus). Briefly, we superposed an oscillatory stress on top of a constant mechanical load - measuring the differential storage modulus $K$ over a wide range of frequencies $(0.01<\omega<10 \mathrm{~Hz})$ and stresses $(0.1<\sigma<8$ Pa). We next fitted the differential storage modulus fitted $K(\sigma, \omega)$ to the following equation:

$$
K^{\prime} \sim \frac{\left(1+\left(\sigma+\sigma_{0, \mathrm{tr}}\right)\right)^{\frac{3}{2}}}{1+\sqrt{\omega_{\text {off }}+\omega}}
$$

where $\omega_{\text {off }}$ is the crosslinker unbinding frequency and $\sigma_{0, t r}$ is the critical stress for stress-stiffening in the fast limit where crosslinkers have not had time to unbind. 


\section{Generation of single-molecule constructs}

Both wild-type a-actinin-4 and the K255E mutant were modified to include a ybbR tag (DSLEFIASKLA) ${ }^{39}$ right after the His $_{6}$-tag. Purified proteins were coupled to Coenzyme A-modified DNA oligonucleotides 20 nucleotides long using a phosphopantetheinyl transferase (SFP synthase)-mediated reaction ${ }^{39}$. A protein-to-DNA molar ratio of 10:1 ensured that only one monomer was coupled to DNA, as evidenced by SDS-PAGE analysis (Extended Data Fig. 3a). Next, 2.5 kilo base pair DNA tethers were PCR-amplified from the pUC19 plasmid (New England Biolabs) with a 5'-biotinylated primer on one side and a 5'-phosphoprimer on the other side. Purification was done with the QIAquick PCR purification kit (Qiagen, Hilden, Germany). The phosphorylated strand was digested using $\lambda$-exonuclease (New England Biolabs) for 2 hours at $37^{\circ} \mathrm{C}$ and purified using an Amicon $30 \mathrm{kDa}$ MWCO filter (Merck, Darmstadt, Germany). Deep Vent exo-DNA polymerase (New England Biolabs) and a 20-nucleotides more upstream primer than the phosphoprimer from the PCR was used to fill up the second DNA strand, creating a 20-nucleotide overhang ${ }^{41}$. This overhang is complementary to the 20-oligonucleotide sequence coupled to the proteins. The generated DNA tether was then ligated to the DNA-protein hybrid by overnight incubation with T4 ligase (New England Biolabs) at room temperature. The stock sample was flash frozen and stored at $-80{ }^{\circ} \mathrm{C}$, and small aliquots were stored at $4{ }^{\circ} \mathrm{C}$ for maximally one week.

\section{Preparation of actin-coated and crosslinker-coated beads}

Unlabeled actin monomers were mixed with biotinylated monomers and fluorescent monomers labeled with Alexa Fluor 647 in a molar ratio of 8:1:1 and polymerized into filaments in $1 \mathrm{~mL}$ F-buffer at a concentration of $2 \mu \mathrm{M}$ for 2 hours. Next, these filaments were mixed with $4 \mu \mathrm{l}$ of $2.4 \mu \mathrm{M}$ Neutravidin-coated beads (NVP-20-5, diameter 2.1 $\mu \mathrm{m}$, Spherotech) and incubated for 15 minutes to couple the filaments to the beads. The actin-coated beads were separated from unbound actin filaments by centrifuging $3 x$ at $1000 \mathrm{rcf}$ for 2 minutes. After every round, $800 \mu \mathrm{l}$ of supernatant was discarded, whilst carefully avoiding disturbing the pellet, and replaced by $800 \mu \mathrm{l}$ of fresh F-buffer. Successful coating was verified using confocal fluorescence microscopy by the presence of a fluorescent ring on the edge of the bead upon excitation with a $638 \mathrm{~nm}$ laser (Extended Data Fig. 3b). For the other bead type, approximately $50 \mathrm{ng}$ of the generated crosslinker-DNA construct was incubated with $2 \mu \mathrm{l}$ NeutrAvidin beads in 10 $\mu \mathrm{l} \mathrm{F}$-buffer for $15 \mathrm{~min}$ in a rotary mixer at $4\left\llcorner{ }^{\circ} \mathrm{C}\right.$, and then rediluted in $\mu \mathrm{l} 500 \mathrm{~F}$-buffer with $100 \mathrm{mM}$ biotin excess to block unbound NeutrAvidin. Unbound biotin was removed during the optical tweezer assay by flushing F-buffer after trapping the beads.

\section{Single molecule data acquisition and analysis}

Force spectroscopy data was collected at $500 \mathrm{~Hz}$ using a custom-built dual trap optical tweezers and a commercial C-Trap (Lumicks). Data was analyzed using custom scripts in Python. The optical traps were calibrated using the power spectrum of the Brownian motion of the trapped beads ${ }^{47}$, obtaining average stiffness values of $\mathrm{K}=0.39 \pm 0.04 \mathrm{pN} \mathrm{nm}^{-1}$. After trapping beads with the two different coatings (Extended Data Fig. 3b), $a$ actinin-4-actin binding was established by approaching and maintaining both beads in close proximity during approximately 10 seconds. Tether lifetime was assessed by rapidly retracting the beads to a set distance - thus increasing the applied force - and measuring the time until the tether broke. To discriminate single from multiple connections, we used the worm-like-chain (WLC) model and the fact that single double-stranded DNA exhibits an overstretching plateau in the force-extension curve at forces above $65 \mathrm{pN}$ (Extended Data Fig. 3c). We pulled on tethers to high forces and observed that the contour length (computed using the WLC) of those that displayed overstretching characteristic of single tethers matched the expected value of $850 \mathrm{~nm}$ within a $\sim 60 \mathrm{~nm}$ range, likely due to the variability in the bead radii and the thickness of the actin coat. Multiple tethers, in contrast to single ones, did not show this characteristic overstretching, and their apparent length was most often shorter (Extended Data Fig. 3c). Therefore, we considered tethers that displayed the expected contour length of $850 \pm 30 \mathrm{~nm}$ and 
bioRxiv preprint doi: https://doi.org/10.1101/2020.07.27.219618; this version posted July 28, 2020. The copyright holder for this preprint (which was not certified by peer review) is the author/funder, who has granted bioRxiv a license to display the preprint in perpetuity. It is made available under aCC-BY 4.0 International license.

373 broke in a clean step. Most tethers showed dissociation below a minute waiting time (55\% of tethers, across all 374 forces). Tethers that lasted longer generally did not break at all, even after several minutes under tension. Hence, 375 lifetimes were determined from tethers showing dissociation within one minute. Lifetime statistical comparisons 376 were obtained by a one-sided t-test. 


\section{References}

1. Marshall, B. T. et al. Direct observation of catch bonds involving cell-adhesion molecules. Nature 423, 190-3 (2003).

2. Liu, B., Chen, W., Evavold, B. D. \& Zhu, C. Accumulation of Dynamic Catch Bonds between TCR and Agonist Peptide-MHC Triggers T Cell Signaling. Cell 157, 357-368 (2014).

3. Doss, B. L. et al. Cell response to substrate rigidity is regulated by active and passive cytoskeletal stress. Proceedings of the National Academy of Sciences 201917555 (2020) doi:10.1073/pnas.1917555117.

4. Thomas, W. E., Trintchina, E., Forero, M., Vogel, V. \& Sokurenko, E. v. Bacterial Adhesion to Target Cells Enhanced by Shear Force. Cell 109, 913-923 (2002).

5. Huang, D. L., Bax, N. A., Buckley, C. D., Weis, W. I. \& Dunn, A. R. Vinculin forms a directionally asymmetric catch bond with F-actin. Science 357, 1-5 (2017).

6. Akiyoshi, B. et al. Tension directly stabilizes reconstituted kinetochore-microtubule attachments. Nature 468, 576-579 (2010).

7. Yamada, A. et al. Catch-bond behaviour facilitates membrane tubulation by nonprocessive myosin 1b. Nature Communications 5, 3624 (2014).

8. Laakso, J. M., Lewis, J. H., Shuman, H. E. \& Ostap, M. E. Myosin I Can Act As a Molecular Force Sensor. Science 321, 133-137 (2008).

9. Mbanga, B. L., lyer, B. V. S., Yashin, V. v. \& Balazs, A. C. Tuning the Mechanical Properties of Polymer-Grafted Nanoparticle Networks through the Use of Biomimetic Catch Bonds. Macromolecules 49, 1353-1361 (2016).

10. Lee, C. -y. et al. Actin depolymerization under force is governed by lysine 113:glutamic acid 195-mediated catch-slip bonds. Proceedings of the National Academy of Sciences 110, 5022-5027 (2013).

11. Yago, T. et al. Platelet glycoprotein Ib $\alpha$ forms catch bonds with human WT vWF but not with type 2B von Willebrand disease vWF. Journal of Clinical Investigation 118, 3195-3207 (2008).

12. Kim, J., Zhang, C.-Z., Zhang, X. \& Springer, T. A. A mechanically stabilized receptorligand flex-bond important in the vasculature. Nature 466, 992-995 (2010).

13. Luo, T., Mohan, K., Iglesias, P. a \& Robinson, D. N. Molecular mechanisms of cellular mechanosensing. Nature materials 12, 1064-71 (2013).

14. Schiffhauer, E. S. et al. Mechanoaccumulative Elements of the Mammalian Actin Cytoskeleton. Current Biology 26, 1473-1479 (2016). 
15. Buckley, C. D. et al. Cell adhesion. The minimal cadherin-catenin complex binds to actin filaments under force. Science 346, 1254211 (2014).

16. Sokurenko, E. v., Vogel, V. \& Thomas, W. E. Catch-Bond Mechanism of Force-Enhanced Adhesion: Counterintuitive, Elusive, but ... Widespread? Cell Host and Microbe 4, 314323 (2008).

17. Sauer, M. M. et al. Catch-bond mechanism of the bacterial adhesin FimH. Nature Communications 7, 10738 (2016).

18. Mould, A. P. et al. Cyclic Mechanical Reinforcement of Integrin - Ligand Interactions. Cell 1060-1068 (2013) doi:10.1016/j.molcel.2013.01.015.

19. Friedland, J. C., Lee, M. H. \& Boettiger, D. Mechanically Activated Integrin Switch Controls 51 Function. Science 323, 642-644 (2009).

20. Luca, V. C. et al. Notch-Jagged complex structure implicates a catch bond in tuning ligand sensitivity. Science 355, 1320-1324 (2017).

21. Burla, F., Mulla, Y., Vos, B. E., Aufderhorst-Roberts, A. \& Koenderink, G. H. From mechanical resilience to active material properties in biopolymer networks. Nature Reviews Physics (2019) doi:10.1038/s42254-019-0036-4.

22. Kaplan, J. M. et al. Mutations in ACTN4, encoding $\alpha$-actinin-4, cause familial focal segmental glomerulosclerosis. Nature Genetics 24, 251-256 (2000).

23. Feng, D. et al. Disease-causing mutation in $\alpha$-actinin-4 promotes podocyte detachment through maladaptation to periodic stretch. Proceedings of the National Academy of Sciences 115, 1517-1522 (2018).

24. Wegst, U. G. K. et al. Bioinspired structural materials. Nature materials 14, 23-36 (2014).

25. Feng, D., DuMontier, C. \& Pollak, M. R. Mechanical challenges and cytoskeletal impairments in focal segmental glomerulosclerosis. American Journal of PhysiologyRenal Physiology 314, F921-F925 (2018).

26. Ehrlicher, A. J. et al. Alpha-actinin binding kinetics modulate cellular dynamics and force generation. Proceedings of the National Academy of Sciences 112, 201505652 (2015).

27. Weins, A. et al. Disease-associated mutant alpha-actinin-4 reveals a mechanism for regulating its F-actin-binding affinity. Proceedings of the National Academy of Sciences of the United States of America 104, 16080-5 (2007).

28. Galkin, V. E., Orlova, A., Salmazo, A., Djinovic-Carugo, K. \& Egelman, E. H. Opening of tandem calponin homology domains regulates their affinity for F-actin. Nature Structural \& Molecular Biology 17, 614-616 (2010). 
29. Ribeiro, E. D. A. et al. The structure and regulation of human muscle $\alpha$-Actinin. Cell 159, 1447-1460 (2014).

30. Yao, N. Y. et al. Stress-Enhanced Gelation: A Dynamic Nonlinearity of Elasticity. Physical Review Letters 110, 018103 (2013).

31. Mulla, Y., Oliveri, G., Overvelde, J. T. B. \& Koenderink, G. H. Crack Initiation in Viscoelastic Materials. Physical Review Letters 120, 268002 (2018).

32. Mulla, Y. \& Koenderink, G. H. Crosslinker mobility weakens transient polymer networks. Physical Review E 98, 062503 (2018).

33. Bell, G. Models for the specific adhesion of cells to cells. Science 200, 618-627 (1978).

34. Mulla, Y., Wierenga, H., Alkemade, C., ten Wolde, P. R. \& Koenderink, G. H. Frustrated binding of biopolymer crosslinkers. Soft Matter 3036-3042 (2019) doi:10.1039/c8sm02429d.

35. Mulla, Y., Mackintosh, F. C. \& Koenderink, G. H. Origin of Slow Stress Relaxation in the Cytoskeleton. Physical Review Letters 122, 218102 (2019).

36. Rosales, A. M. \& Anseth, K. S. The design of reversible hydrogels to capture extracellular matrix dynamics. Nature Reviews Materials 1, 15012 (2016).

37. Yeom, B. et al. Abiotic tooth enamel. Nature 543, 95-98 (2017).

38. Garcia-Manyes, S., Liang, J., Szoszkiewicz, R., Kuo, T.-L. \& Fernández, J. M. Forceactivated reactivity switch in a bimolecular chemical reaction. Nature chemistry 1 , 236-242 (2009).

39. Yin, J. et al. Genetically encoded short peptide tag for versatile protein labeling by Sfp phosphopantetheinyl transferase. Proceedings of the National Academy of Sciences 102, 15815-15820 (2005).

40. Pollard, T. D., Blanchoin, L. \& Mullins, R. D. Molecular Mechanisms Controlling Actin Filament Dynamics in Nonmuscle Cells. Annual Review of Biophysics and Biomolecular Structure 29, 545-576 (2000).

41. Avellaneda, M. J., Koers, E. J., Minde, D. P., Sunderlikova, V. \& Tans, S. J. Simultaneous sensing and imaging of individual biomolecular complexes enabled by modular DNAprotein coupling. Communications Chemistry 3, 20 (2020).

42. Smith, S. B., Cui, Y. \& Bustamante, C. Overstretching B-DNA: The Elastic Response of Individual Double-Stranded and Single-Stranded DNA Molecules. Science 271, 795-799 (1996).

43. Gardel, M. L. et al. Elastic behavior of cross-linked and bundled actin networks. Science (New York, N.Y.) 304, 1301-5 (2004). 
44. Tang, V. W. \& Brieher, W. M. alpha-Actinin-4/FSGS1 is required for Arp2/3-dependent actin assembly at the adherens junction. Journal of Cell Biology 196, 115-130 (2012).

45. Mulla, Y., Wierenga, H., Alkemade, C., ten Wolde, P. R. \& Koenderink, G. H. Frustrated binding of biopolymer crosslinkers. Soft Matter 15, (2019).

46. Schneider, C. A., Rasband, W. S. \& Eliceiri, K. W. NIH Image to ImageJ: 25 years of image analysis. Nature Methods 9, 671-675 (2012).

47. Berg-Sørensen, K. \& Flyvbjerg, H. Power spectrum analysis for optical tweezers. Review of Scientific Instruments 75, 594-612 (2004).

Author contributions: Y.M. and G.H.K. conceived and designed the study. M.J.A. and S.J.T. designed the optical tweezer experiments. M.J.A., A.R. and L.B. performed the optical tweezer experiments. M.J.A. and A.R. analyzed the optical tweezer experiments. Y.M. performed and analyzed all other experiments and designed and simulated the theoretical model. Y.M., M.J.A, S.J.T. and G.H.K. wrote the manuscript with input from A.R. and L.B. All authors approved the final version.

Acknowledgements: We thank Martin van Hecke and Celine Alkemade for critical reading of the manuscript. We thank Taeyoon Kim, Pieter Rein ten Wolde, Kees Storm, Wouter Ellenbroek, Chase Broedersz, David Brueckner and Mareike Berger for fruitful discussions. We thank William Brieher and Vivian Tang for the kind gift of purified a-actinin-4 (wild type and the K255E point mutant) and their plasmids, Marjolein Kuit-Vinkenoog for actin and further purification a-actinin-4 and Vanda Sunderlíková for design, mutagenesis, cloning and purifying of the a-actinin- 4 constructs used in the single molecule experiments. This work is part of the research program of the Netherlands Organization for Scientific Research (NWO). We gratefully acknowledge financial support from an ERC Starting Grant (335672MINICELL) awarded to G.K.

Data availability: The data that support the findings of this study are available from the corresponding authors upon reasonable request.

Code availability: Custom-written scripts used in this study are available from the corresponding authors upon reasonable request.

\section{Additional information}

Supplementary Information is available for this paper.

Correspondence and requests for materials should be addressed to G.H.K and S.J.T. 
bioRxiv preprint doi: https://doi.org/10.1101/2020.07.27.219618; this version posted July 28, 2020. The copyright holder for this preprint (which was not certified by peer review) is the author/funder, who has granted bioRxiv a license to display the preprint in perpetuity. It is made available under aCC-BY 4.0 International license. 


\section{Supplementary Information}

\section{Weak catch bonds make strong networks}

Yuval Mulla ${ }^{1,2}$, Mario J Avellaneda ${ }^{1}$, Antoine Roland ${ }^{1}$, Lucia Baldauf ${ }^{1,3}$, Sander J Tans ${ }^{1,3 *}$, Gijsje H Koenderink $^{1,3 *}$

${ }^{1}$ Living Matter Department, AMOLF, Amsterdam, The Netherlands

${ }^{2}$ Institute for Biological Physics, University of Cologne, Cologne, Germany

${ }^{3}$ Department of Bionanoscience, Kavli Institute of Nanoscience Delft, Delft University of Technology, Delft $2629 \mathrm{HZ}$, the Netherlands

*Correspondence to: g.h.koenderink@tudelft.nl, tans@amolf.nl

\section{Frequency-dependent rheology shows that crosslinking affects the dynamics, but not the structure of actin networks}

To test for the influence of the binding affinity of the two $\alpha$-actinin- 4 variants (wild type and $\mathrm{K} 255 \mathrm{E}$ ) on the dynamics of actin networks, we performed small-amplitude oscillatory shear measurements on the crosslinked actin networks over a range of oscillation frequencies $\left(10^{-3}\right.$ to $10 \mathrm{~Hz}$, see Extended Data Fig. 4a). For both variants, the frequency spectrum of the shear moduli has a functional form that is characteristic of transiently crosslinked semiflexible polymer networks ${ }^{1,2}$. For low frequencies, below the crosslinker unbinding frequency, both networks deform viscoelastically, with the storage modulus $G$ ' and loss modulus G' both following a power law dependence on frequency with an exponent of $1 / 2$. This exponent arises from the superposition of multiple relaxation times of many crosslinkers connecting a single filament to the surrounding network, which collectively cause a $\omega^{-1 / 2}$ relaxation ${ }^{1}$. We observe a characteristic relaxation frequency where G' exhibits a peak and G' exhibits an elastic plateau. This relaxation frequency corresponds to the crosslinker unbinding rate ${ }^{1}$. At frequencies above $5 \mathrm{~Hz}$, both moduli show a slight upturn, which reflects the influence of viscous drag on the actin filaments.

When we compare the frequency spectrum for actin networks crosslinked with the two $\alpha$ actinin-4 variants, we observe that replacing the wild type variant by the K255E mutant causes a shift of the relaxation frequency, indicating slower unbinding as expected from the higher binding affinity. When we normalize the applied frequency with the relaxation frequency, the G' and G" curves for the K255E mutant can be super-imposed on the wild 
type $\alpha$-actinin- 4 curves with only a small deviation at high normalized frequencies, which we attribute to the effect of the viscous drag on the filaments (Extended Data Fig. 4b).

We can tune the relaxation frequency for actin networks crosslinked with K255E by increasing the temperature ${ }^{3}$ from $10{ }^{\circ} \mathrm{C}$ to $25^{\circ} \mathrm{C}$ such that it equals the peak frequency of networks crosslinked with the wild type variant at low temperature $\left(10^{\circ} \mathrm{C}\right)$. Now, the timedependent linear rheology of both networks is indistinguishable even without normalizing the frequency (Extended Data Fig. 4c). This collapse suggests that, although the crosslinker lifetime is longer for the wild type a-actinin-4 than for the K255E mutant, the network structure is not significantly different. For example, any change in the typical crosslinker distance would have altered the shear modulus ${ }^{1}$. Indeed, with light microscopy we do not find any bundles, indicating that both networks are isotropically crosslinked (Extended Data Fig. 8).

\section{Catch bonds still enhance network strength when taking into account their dimeric molecular structure}

In the model presented in the main text, crosslinkers are either bound or unbound. However, the $\alpha$-actinin- 4 crosslinkers in the experiments can also be partially bound (i.e., only to one actin filament), because they have two independent actin-binding domains separated by a spacer. To test whether allowing for partially bound crosslinkers qualitatively affects the model predictions, we expand the model to allow for three binding states, where doubly bound crosslinkers become singly bound crosslinkers with an unbinding rate of $k_{2 \rightarrow 1}\left(f_{i}\right)$, following force-induced unbinding according to Eq. (1) of the Material and Methods. Singly bound crosslinkers become fully unbound with a rate of $k_{1 \rightarrow 0}$, or doubly bound with a rate of $k_{1 \rightarrow 2}$. Force is only shared between neighboring doubly-bound crosslinkers. For simplicity, we have chosen $k_{1 \rightarrow 0}=k_{2 \rightarrow 1}(0)$ and $k_{0 \rightarrow 1}=k_{1 \rightarrow 2}=1$. Importantly, allowing for this additional, partially bound state does not qualitatively affect the predictions of the computational model, as catch bonds still form networks that are both stronger and more dynamic than networks formed with slip bonds (Extended Data Fig. 6d). For all other simulations, we therefore use a two-state model as it is the most minimal model that captures our experimental results.

\section{Catch bonds enhance the strength of materials that fracture via crack propagation}

Our experiments demonstrate that catch bonds collectively provide stronger networks, even though they are actually weaker on the single molecule level. This raises the question how many bonds are required for the catch bond advantage to emerge. To answer this question, we performed fracturing simulations at different network sizes (1-200 linkers, Extended Data Figure 7a,b). We find that for both catch and slip bond networks, the rupture strength and 
bond turnover number initially increase with increasing bond number, peak when the bond number reaches around 10, and then continuously decrease with increasing bond number. Remarkably, slip bond networks are stronger for systems smaller than 10 bonds, while catch bond networks have both larger network strength and bond turnover for networks larger than 10 bonds.

Why is catch bonding (only) superior in large networks? To understand this, we need the notion of a critical crack size from fracture mechanics ${ }^{4,5}$. When a large crack (a part of the network that is devoid of crosslinkers) is under stress, bonds at the edge of the crack rapidly unbind, causing crack propagation and eventually network fracturing. However, when the crack is still small, linker binding may heal the crack before it spreads. Therefore, there is a critical crack size that is on the verge of becoming unstable. For systems below this critical crack size, the network strength increases with the bond number as it becomes increasingly unlikely that all linkers simultaneously unbind. For large systems however, increasing the network size allows for more locations at which a crack can be initiated - causing a decrease in network strength. Combined, these two effects explain why we observe a biphasic dependence of network strength on system size, with a cross-over at a critical crack size (Extended Data Fig. 6a,b, see Refs. ${ }^{5,6}$ for a more detailed and quantitative explanation).

So why are catch bonds only effective for systems larger than the critical crack size? Catch bonds rely on bond redistribution to enhance the network strength (Extended Data Fig. 6c). The 'dissociation-on-demand' of unforced catch bonds increases the pool of unbound crosslinkers and thereby crosslinker binding in the entire network, causing a net crosslinker migration from stress-free to high-stress areas, as can be seen from the narrow force distribution (Fig. 3a). This increased binding in high-stress areas makes cracks less prone to becoming unstable (Fig. 3b), thus increasing the network strength. However, this effect relies on catch bonds to migrate from outside of the critical crack size towards the crack, and therefore only emerges in networks larger than the critical crack size.

Although it is difficult to precisely know the critical crack size for viscoelastic materials, any macroscopic network that can be studied with bulk rheology techniques is orders of magnitude above this size threshold ${ }^{5}$. Furthermore, laser ablation experiments have revealed that cells are also significantly larger than the critical crack size ${ }^{7}$. Therefore, we conclude that the catch bond advantage is general and should emerge both in cells and macroscopic networks. Lastly, as cell adhesion typically also relies on hundreds of linkers ${ }^{8}$, catch bonds likely also strengthen cell-cell and cell-matrix adhesions whilst simultaneously facilitating sliding. Therefore, our results could also explain why biological adhesins are very often catch bonds ${ }^{9}$. 


\section{References}

1.7 Broedersz, C. P. et al. Cross-Link-Governed Dynamics of Biopolymer Networks. Physical Review Letters 105, 238101 (2010).

2.9 Mulla, Y., Mackintosh, F. C. \& Koenderink, G. H. Origin of Slow Stress Relaxation in the Cytoskeleton. Physical Review Letters 122, 218102 (2019).

3.1 Mulla, Y., Wierenga, H., Alkemade, C., ten Wolde, P. R. \& Koenderink, G. H. Frustrated binding of biopolymer crosslinkers. Soft Matter 3036-3042 (2019) doi:10.1039/c8sm02429d.

4.3 Griffith, A. A. The Phenomena of Rupture and Flow in Solids. Philosophical Transactions of the Royal Society A: Mathematical, Physical and Engineering Sciences 221, 163-198 (1921).

5.6 Mulla, Y., Oliveri, G., Overvelde, J. T. B. \& Koenderink, G. H. Crack Initiation in Viscoelastic Materials. Physical Review Letters 120, 268002 (2018).

68 Mulla, Y. \& Koenderink, G. H. Crosslinker mobility weakens transient polymer networks. Physical Review E 98, 062503 (2018).

7.0 Tinevez, J.-Y. et al. Role of cortical tension in bleb growth. Proceedings of the National Academy of Sciences 106, 18581-18586 (2009).

8. Truong Quang, B.-A., Mani, M., Markova, O., Lecuit, T. \& Lenne, P.-F. Principles of ECadherin Supramolecular Organization In Vivo. Current Biology 23, 2197-2207 (2013).

94 Sokurenko, E. v., Vogel, V. \& Thomas, W. E. Catch-Bond Mechanism of Force-Enhanced Adhesion: Counterintuitive, Elusive, but ... Widespread? Cell Host and Microbe 4, 314-323 (2008). 


\section{Extended Data Figures}

\section{Weak catch bonds make strong networks}

Yuval Mulla ${ }^{1,2}$, Mario J Avellaneda ${ }^{1}$, Antoine Roland ${ }^{1}$, Lucia Baldauf ${ }^{1,3}$, Sander J Tans ${ }^{1,3 \star}$, Gijsje H Koenderink ${ }^{1,3 *}$

${ }^{1}$ Living Matter Department, AMOLF, Amsterdam, The Netherlands

${ }^{2}$ Institute for Biological Physics, University of Cologne, Cologne, Germany

${ }^{3}$ Department of Bionanoscience, Kavli Institute of Nanoscience Delft, Delft University of Technology, Delft $2629 \mathrm{HZ}$, the Netherlands

*Correspondence to: g.h.koenderink@tudelft.nl, tans@amolf.nl 
bioRxiv preprint doi: https://doi.org/10.1101/2020.07.27.219618; this version posted July 28, 2020. The copyright holder for this preprint (which was not certified by peer review) is the author/funder, who has granted bioRxiv a license to display the preprint in perpetuity. It is made available under aCC-BY 4.0 International license.

a
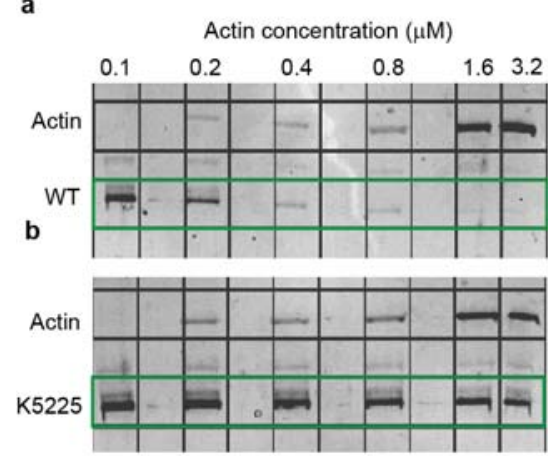

c

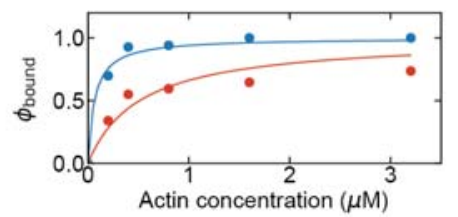

d

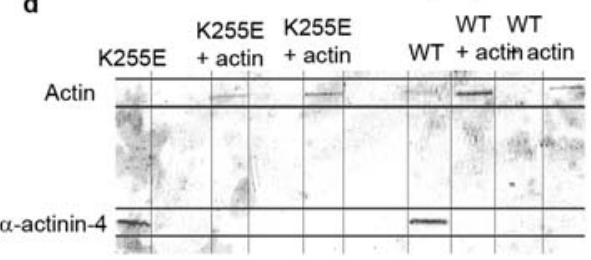

Extended Data Fig. 1: High-speed co-sedimentation measurements of the affinity of $\alpha$-actinin-4 (WT) and K255E crosslinkers for actin filaments. a, b, supernatant resulting from a high-speed centrifugation of a mixture of actin filaments and crosslinkers was run on an SDS-page gel. The bands on the bottom show the aactinin-4 (WT) or K255E (resp. $\mathbf{a}$ and $\mathbf{b}$, molecular weight $\sim 100 \mathrm{kDa}$ in both cases), while the bands on the top show actin (42 kDa). Each labeled column contained a different actin concentration as indicated. Some lanes were kept empty as spacers. The crosslinker concentration was fixed at $0.1 \mu \mathrm{M}$. c, The fraction of bound crosslinkers, as determined from the co-sedimentation assay, as a function of the actin concentration was fit to the equation: $\varphi_{\text {bound }}={ }^{C_{\text {actin }}} / K_{a}$, where $K_{a}$ is the affinity of the crosslinker. $\mathbf{d}$, Consistent with the high affinity of both crosslinkers, SDS-page gels of supernatant resulting from a high-speed centrifugation of a crosslinked actin network at the concentration used in all our experiments ( $48 \mu \mathrm{M}$ actin together with $0.48 \mu \mathrm{M}$ crosslinker) does not show any measurable fraction of unbound crosslinkers. 
bioRxiv preprint doi: https://doi.org/10.1101/2020.07.27.219618; this version posted July 28, 2020. The copyright holder for this preprint (which was not certified by peer review) is the author/funder, who has granted bioRxiv a license to display the preprint in perpetuity. It is made available under aCC-BY 4.0 International license.
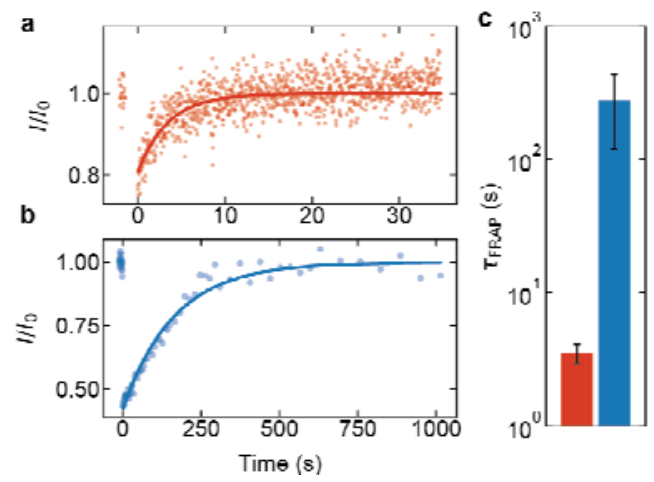

Extended Data Fig. 2: Fluorescence recovery after photobleaching measurements reveal that $\alpha$-actinin-4 crosslinkers are more dynamic than the K255E mutant. Example fluorescence recovery curves of a-actinin-4 (a) and K255E (b) in the presence of $48 \mu \mathrm{M}$ actin show full recovery of both proteins after photobleaching at time $\mathrm{t}=0$, but with different timescales. The solid lines represent exponential fits to the data (see Methods). c, Average recovery time for a-actinin-4 (red) and for K255E (blue), with the standard error on basis of 6 repeats per condition. Measurements were performed at $25^{\circ} \mathrm{C}$. 
bioRxiv preprint doi: https://doi.org/10.1101/2020.07.27.219618; this version posted July 28, 2020. The copyright holder for this preprint (which was not certified by peer review) is the author/funder, who has granted bioRxiv a license to display the preprint in perpetuity. It is made available under aCC-BY 4.0 International license.

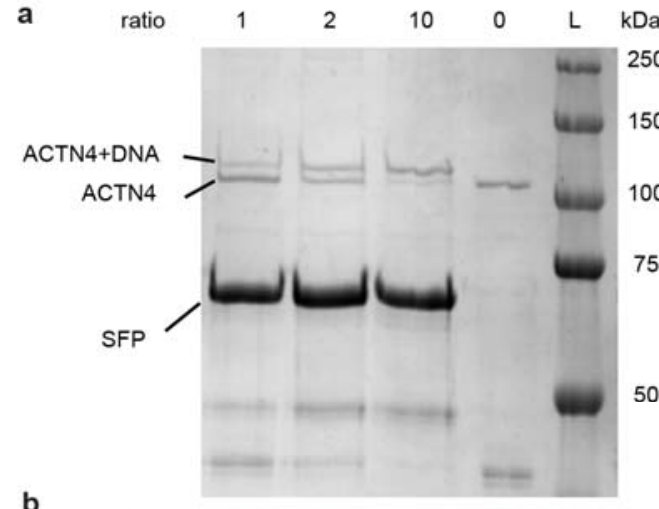

b

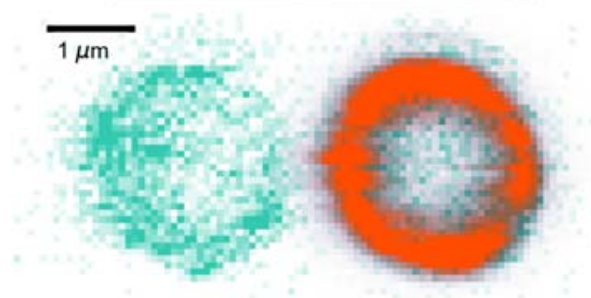

C

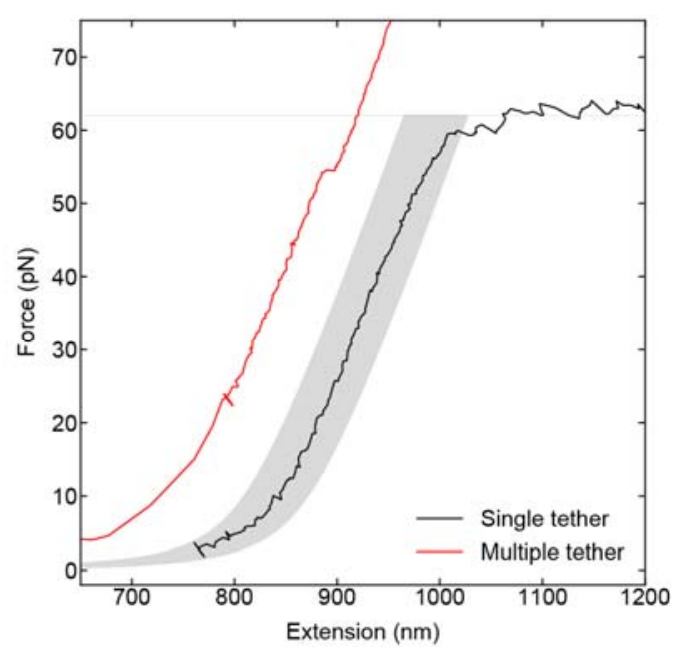

Extended Data Fig. 3: Generation and classification of $\alpha$-actinin-4/actin tethers. a, DNA was coupled to aactinin-4 (WT or K255E) using an SFP synthase-mediated reaction. Because a-actinin-4 is a homodimer, the $\mathrm{yBBr}$ tag used for coupling is present in both monomers. To favour DNA attachment to only one monomer, we performed coupling reactions with several DNA titrations, and the coupling yields were quantified using SDSPAGE gel electrophoresis. The DNA:a-actinin-4 molar ratios are indicated above each lane. At a molar ratio of $1: 1$, most of the a-actinin-4 is uncoupled, i.e. most dimers will be either not coupled or have only one monomer coupled to DNA. b, Concurrent confocal fluorescence images of a trapped bead coated with a-actinin-4 (left) and a trapped bead coated with actin filaments (right). The bead's autofluorescence is depicted in green, and the fluorescent emission of Alexa Fluor 647-tagged actin is depicted in orange. c, Force-extension curves showing the overstretching regime of a single dsDNA tether (black), and a case where the two beads are linked by multiple tethers, which yields a shorter contour length and higher forces without unzipping (red). Variability in bead radii and actin layer thickness results in force-extension curves that can be shifted along the Extension axis, from the theoretical $850 \mathrm{~nm}$ by $\pm 30 \mathrm{~nm}$. Grey area: "single-tether region". Tethers with a force-extension curve within this area that broke in a single step were regarded as single tethers and hence included in measuring the forcedependent lifetime. 
bioRxiv preprint doi: https://doi.org/10.1101/2020.07.27.219618; this version posted July 28, 2020. The copyright holder for this preprint (which was not certified by peer review) is the author/funder, who has granted bioRxiv a license to display the preprint in perpetuity. It is made available under aCC-BY 4.0 International license.
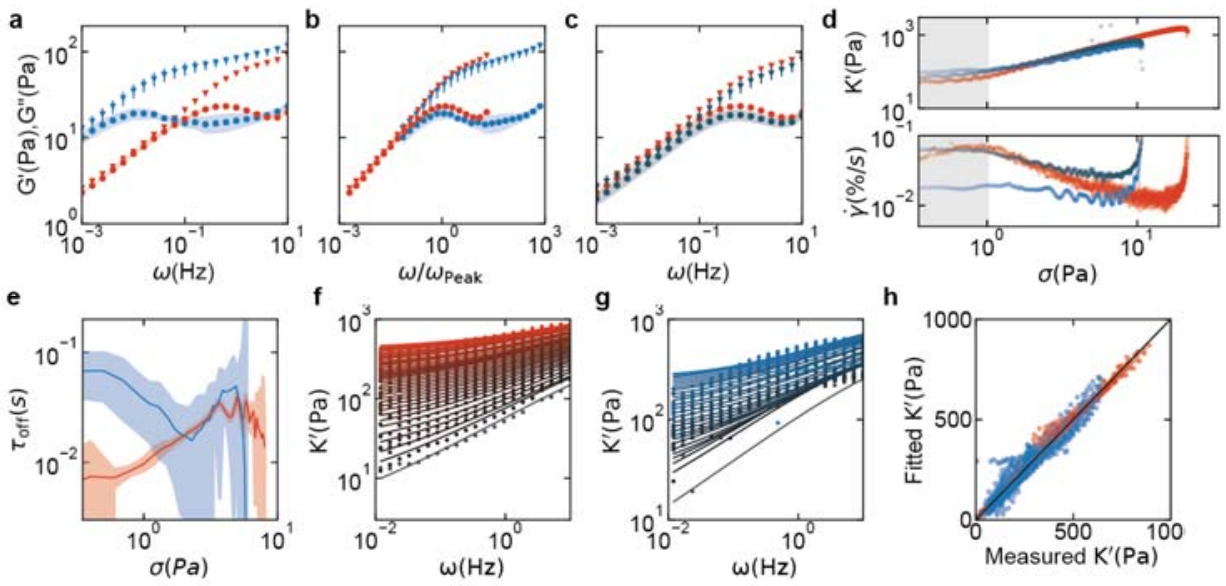

Extended Data Fig. 4: Nonlinear and temperature-dependent rheology of actin networks crosslinked by $\alpha$ actinin-4 or K255E. a-c, The storage (triangles) and loss moduli (circles) were measured using small amplitude oscillatory shear. The moduli are shown as a function of frequency (a) and as a function of the frequency normalized by the frequency at which the loss modulus peaks (b). The peak frequency is $0.5 \mathrm{~Hz}$ for a-actinin-4 (red), and $0.01 \mathrm{~Hz}$ for the K255E mutant (light blue). Both curves are measured at $10^{\circ} \mathrm{C}$. c. The time-dependent rheology of actin networks is compared between a-actinin-4 crosslinking at $10^{\circ} \mathrm{C}$ (red) and K255E crosslinking at $25^{\circ} \mathrm{C}$ (dark blue). The standard error indicated by bars and shaded regions is on basis of 4 repeats per condition. The collapses in $\mathbf{b}$ and $\mathbf{c}$ show that the crosslinker unbinding kinetics, but not the network structure, is significantly different for the different conditions (see Main Text). $\mathbf{d}$, Representative example curves of the differential storage modulus at $0.5 \mathrm{~Hz}$ (top) and of the strain rate (bottom) are plotted against the applied shear stress for actin networks crosslinked by a-actinin- $4 \mathrm{WT}$ at $10{ }^{\circ} \mathrm{C}$ (red), K255E at $10{ }^{\circ} \mathrm{C}$ (light blue) or K255E at $25^{\circ} \mathrm{C}$ (dark blue). We define the rupture strain as the data point where $K^{\prime}$ peaks. e-h, We apply a semiflexible polymer network model to fit the frequency-dependent differential elastic modulus as a function of prestress (see Methods). e, Thus, we extract the crosslinker bound lifetime as a function of stress for both a-actinin-4 (red) and the K255E mutant (blue) at $25^{\circ} \mathrm{C}$. The shaded areas represent the error on basis of the fits. The bound lifetime of the mutant is significantly longer at low stress, but the lifetimes of catch and slip bonds become similar at high stress as the bound lifetime of the catch bonds increases. The abrupt decay of bound lifetime in the K255E-crosslinked network when the stress reaches $5 \mathrm{~Pa}$ is due to network fracturing. $\mathbf{h}$, the fitted $\mathrm{K}^{\prime}$ shows quantitative agreement with the measured $\mathrm{K}^{\prime}$ for both catch and slip bonds. 
bioRxiv preprint doi: https://doi.org/10.1101/2020.07.27.219618; this version posted July 28, 2020. The copyright holder for this preprint (which was not certified by peer review) is the author/funder, who has granted bioRxiv a license to display the preprint in perpetuity. It is made available under aCC-BY 4.0 International license.
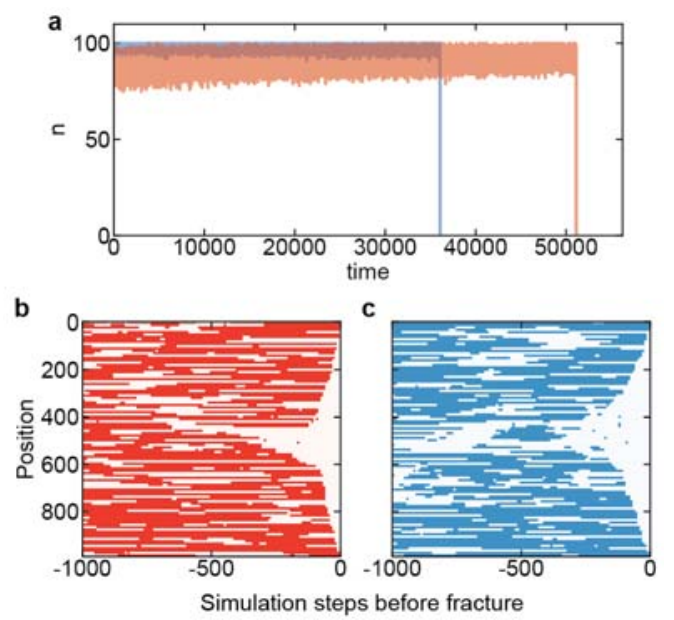

Extended Data Fig. 5: Example simulation runs. a, Time trace of the bound number of catch bonds (red) and slip bonds (blue) in a network undergoing a linearly increasing stress (see Extended Data Table 1 for parameters). As the catch bonds have faster dynamics than the slip bonds, a larger spread in the bound fraction is observed. After a long time of steady state fluctuations, the networks suddenly fracture as the number of linkers rapidly goes to 0 . b, c, Kymographs showing at which positions there are bonds (red for catch bonds, blue for slip bonds) or no bonds (white). At steady state, linkers continuously bind and unbind ( -1000 to approximately -300 steps). Cracks can spontaneously initiate and propagate through the network (the last $\sim 300$ steps of the simulation) for both catch and slip bonds in a similar manner. 
bioRxiv preprint doi: https://doi.org/10.1101/2020.07.27.219618; this version posted July 28, 2020. The copyright holder for this preprint (which was not certified by peer review) is the author/funder, who has granted bioRxiv a license to display the preprint in perpetuity. It is made available under aCC-BY 4.0 International license.

\begin{tabular}{|c|c|c|c|c|c|}
\hline Figure(s) & $\boldsymbol{\sigma}$ & $\boldsymbol{\sigma}$ & $\boldsymbol{N}$ & $\boldsymbol{k}_{\mathbf{1}}$ & $\boldsymbol{k}_{\mathbf{2}}$ \\
\hline Fig. 2d, Extended Data Fig. 6c-d, 8 & NA & $10^{-5}$ & 100 & $10^{-2}$ & $10^{-1}$ \\
\hline Fig. 3a-b & 0.3 & NA & $10^{3}$ & $10^{-2}$ & $10^{-1}$ \\
\hline Extended Data Fig. 6a-b & NA & $10^{-5}$ & $1-100$ & $10^{-2}$ & $10^{-1}$ \\
\hline Extended Data Fig. 7a & NA & $10^{-5}$ & 100 & $10^{-2}-10^{1}$ & $10 * k_{1}$ \\
\hline
\end{tabular}

Extended Data Table 1: Table of parameters used for network simulations. In all simulations, we use $k_{o n}=$ $1, f_{1 / e}^{\text {slip }}=1$ and $f_{1 / e}^{\text {catch }}=0.5$. The applied stress $(\sigma)$, stress rate $(\dot{\sigma})$, number of crosslinkers $(N)$ and bond affinities $\left(k_{2}\right.$ and $\left.k_{2}\right)$ varied for different simulations as shown in this table. For catch bonds (red in all grraphs) and strong (low temperature) slip bonds (dark blue in all graphs), we used $k_{\mathrm{off}, 0}^{\text {slip }}=k_{1}$. For weak (high temperature) slip bonds (light blue in all graphs), we use $k_{\text {off }, 0}^{\text {slip }}=k_{2}$. Lastly, $k_{\text {off }, 0}^{\text {catch }}=k_{2}$ for catch bonds and $k_{\text {off }, 0}^{\text {catch }}=0$ for both strong and weak slip bonds. NA stands for 'Not Applicable'. All units are dimensionless as explained in the methods. 
bioRxiv preprint doi: https://doi.org/10.1101/2020.07.27.219618; this version posted July 28, 2020. The copyright holder for this preprint (which was not certified by peer review) is the author/funder, who has granted bioRxiv a license to display the preprint in perpetuity. It is made available under aCC-BY 4.0 International license.
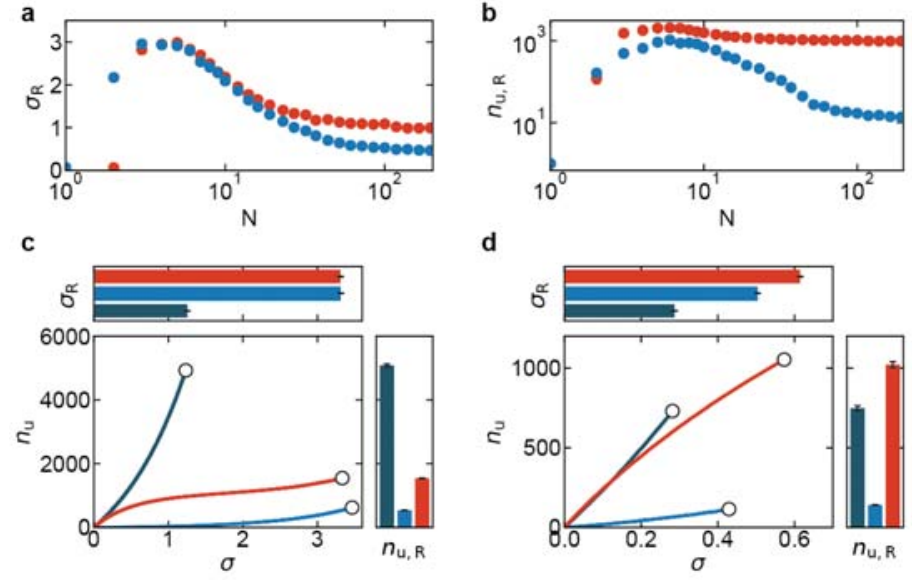

Extended Data Fig. 6: Simulations show that catch bonds only provide a mechanical advantage over slip bonds when they are mobile and present in sufficiently large numbers. The system size dependence of the rupture stress (a) and bond turnover at the point of rupture (b) reveals that catch bonds (red) are only stronger than slip bonds (blue) for networks larger than $\sim 10$ bonds, emphasizing that the increased network strength by catch bonding is an emergent property (Supplementary Information). Each data point is the average of 10 repeats and the standard errors are smaller than the symbol size. c, Catch bond-induced network strengthening is not observed when crosslinkers are immobile and rebind in the same location from which they unbound. The bond turnover as a function of stress reveals catch bonds (red) cause more dynamic materials (right), but do not enhance strength (top) compared to strong slip bonds (light blue) and are less dynamic than networks consisting of weak slip bonds (dark blue). The error bars represent the standard error on basis of 10 repeats per condition. d, We also considered a three-state model where linkers are doubly bound, singly bound or unbound (Supplementary Information "Three-state model"). Similar to the two-state model, the bond turnover as a function of stress reveals that networks of catch bonds (red) are stronger and more deformable than networks of strong slip bonds (light blue) or weak slip bonds (dark blue). 
bioRxiv preprint doi: https://doi.org/10.1101/2020.07.27.219618; this version posted July 28, 2020. The copyright holder for this preprint (which was not certified by peer review) is the author/funder, who has granted bioRxiv a license to display the preprint in perpetuity. It is made available under aCC-BY 4.0 International license.

a

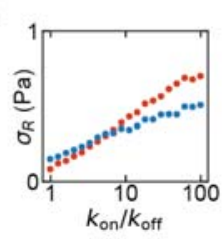

b

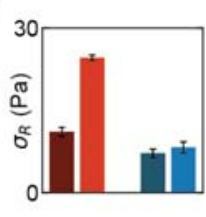

Extended Data Fig. 7: Catch bonding is only effective when the binding rate is high. a, Simulations of the rupture stress as a function of the binding rate $k_{\mathrm{on}} / k_{\mathrm{of}, 0}^{\mathrm{slip}}$, keeping $k_{\mathrm{of}, 0}^{\text {slip }} / k_{\mathrm{off}, 0}^{\text {catch }}$ fixed (see Methods and Extended Data Table 1), shows that catch bonds (red) are only stronger than slip bonds (blue) when the binding rate is high. b, Enhancing the bond lifetime in experiments by decreasing the temperature from $25^{\circ} \mathrm{C}$ (light) to $10{ }^{\circ} \mathrm{C}$ (dark) increases the rupture stress more steeply for wild type a-actinin-4 (red) than for K255E (blue) as the catch bond advantage relies on bond redistribution and therefore on the binding rate (Main Text). 
bioRxiv preprint doi: https://doi.org/10.1101/2020.07.27.219618; this version posted July 28, 2020. The copyright holder for this preprint (which was not certified by peer review) is the author/funder, who has granted bioRxiv a license to display the preprint in perpetuity. It is made available under aCC-BY 4.0 International license.
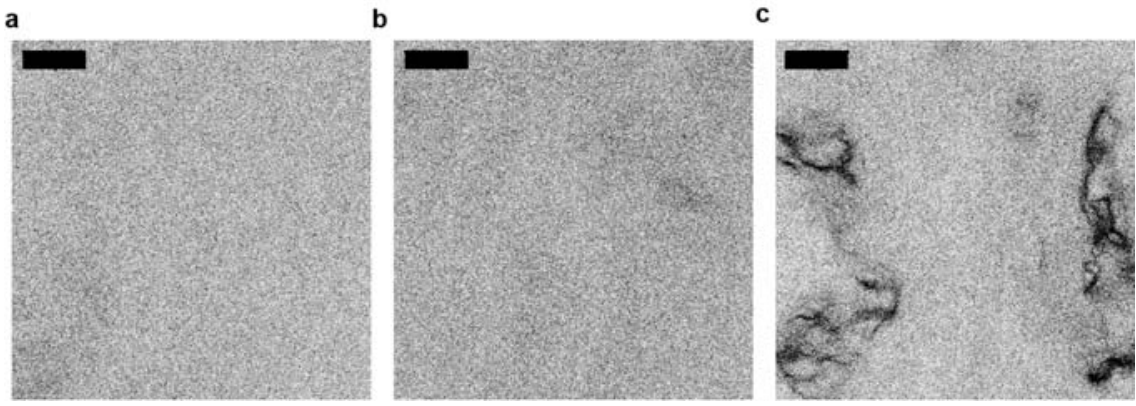

Extended Data Fig. 8: Confocal fluorescence images of crosslinked actin networks. At a 1:100 crosslinker:actin molar ratio, the actin networks studied in this work are isotropic and spatially uniform, for both wild type (a) and K255E a-actinin-4 (b). We do not observe any discernable structure because the mesh size is $\sim 200 \mathrm{~nm}$, which is on the order of the diffraction limit, indicating that filaments are isotropically crosslinked rather than bundled. c, For comparison, actin bundle clusters were observed at a 1:25 a-actinin-4:actin molar ratio. The color coding was inverted for all images to improve the visual contrast between bundles and background. Scale bars are $20 \mu \mathrm{m}$. 